\title{
From the Margins to the Centre: Defining New Mission and Vision for HCI Research in South Asia
}

\author{
Pranjal Jain \\ theUXWhale, India \\ pranjal_jain@live.com \\ Nilavra Bhattacharya \\ University of Texas at Austin, USA \\ nilavra@ieee.org \\ Dhruv Jain \\ University of Washington, Seattle, \\ USA \\ djain@cs.washington.edu \\ Lubna Razaq \\ Information Technology University, \\ Lahore, Pakistan \\ lubna.razaq@itu.edu.pk \\ Mohit Jain \\ Microsoft Research, India \\ mohja@microsoft.com
}

\author{
Samia Ibtasam \\ University of Washington, Seattle, \\ USA \\ samiai@cs.washington.edu
}

Anupriya Tuli

IIIT-Delhi, India

anupriyat@iiitd.ac.in

Rucha Tulaskar

Continuum Managed Services, India

rucha.shende@gmail.com

Rahat Jahangir Rony

North South University, Bangladesh rahatjahangirrony@gmail.com

Suleman Shahid

Lahore University of Management

Science, Lahore, Pakistan

suleman.shahid@lums.edu.pk

\author{
Sumita Sharma \\ University of Oulu, Finland \\ sumita.sharma@oulu.fi
}

Dilrukshi Gamage

University of Moratuwa, Sri Lanka

dilrukshi.gamage@gmail.com

Priyank Chandra

University of Toronto, Canada

priyank.chandra@utoronto.ca
Deepak Ranjan Padhi
Indian Institute of Technology,
Bombay, India
deepak.padhi@iitb.ac.in

Nova Ahmed

North South University, Bangladesh nova.ahmed@northsouth.edu

\author{
Devanuj Balkrishan \\ JK Lakshmipat University, India \\ devanuj@gmail.com
}

\author{
Pushpendra Singh \\ IIIT-Delhi, India \\ psingh@iiitd.ac.in
}

\begin{abstract}
The past two decades have seen an increase in the amount of research in the $\mathrm{CHI}$ community from South Asia with a focus on designing for the unique and diverse socio-cultural, political, infrastructural, and geographical background of the region. However, the studies presented to the $\mathrm{CHI}$ community primarily focus on working with and unpacking the regional contextual constraints (of the users and the infrastructures), thus taking a developmental stance. In this online workshop, we aim to broaden the perspective of the $\mathrm{CHI}$ research and community towards the contributions from the region including and beyond development, by bringing together researchers, designers, and practitioners working or are interested in working within these regions on diverse topics such as universal education, global healthcare, accessibility, sustainability, and more. Through the workshop discussion, group design activity, and

Permission to make digital or hard copies of part or all of this work for personal or classroom use is granted without fee provided that copies are not made or distributed for profit or commercial advantage and that copies bear this notice and the full citation on the first page. Copyrights for third-party components of this work must be honored. For all other uses, contact the owner/author(s).

CHI '21 Extended Abstracts, May 8-13, 2021, Yokohama, Japan

(C) 2021 Copyright held by the owner/author(s).

ACM ISBN xxx-x-xxxx-xxxx- $\mathrm{x}$

https://doi.org/10.1145/Xxxxxxx.xxxxxxx
\end{abstract}

brainstorming, we aim to provide a space for symbiotic knowledge sharing, and defining shared visions and missions for HCI activities in South Asia for including and moving beyond the development agenda.

\section{KEYWORDS}

HCI, South Asia, Beyond development, Vision building

\section{ACM Reference Format:}

Pranjal Jain, Samia Ibtasam, Sumita Sharma, Nilavra Bhattacharya, Anupriya Tuli, Dilrukshi Gamage, Dhruv Jain, Rucha Tulaskar, Priyank Chandra, Lubna Razaq, Rahat Jahangir Rony, Deepak Ranjan Padhi, Mohit Jain, Suleman Shahid, Nova Ahmed, Devanuj Balkrishan, and Pushpendra Singh. 2021. From the Margins to the Centre: Defining New Mission and Vision for HCI Research in South Asia. In CHI Conference on Human Factors in Computing Systems Extended Abstracts (CHI'21 Extended Abstracts), May 8-13, 2021, Yokohama, Japan. ACM, New York, NY, USA, 6 pages. https://doi.org/10.1145/xxxxxxx.xxxxxxxx

\section{BACKGROUND:}

Over the years, the global Human-Computer Interaction (HCI) community has witnessed a plethora of work from South Asia [5]. The focus of the HCI work in the region has been primarily on the Information and Communication Technology (ICT) and its 
developmental implications on users, including those pertaining to the socio-cultural norms, lack of resources, literacy and income (e.g., $[5,6,12,14,17,23,26])$. Some of the conducted research has been initiated and conducted by or in collaboration with researchers from the Global North (e.g., [2, 10, 18, 22, 24]), focusing primarily on challenges of the under-served user groups. However, the South Asian region has more to offer, and needs to be looked at more than merely as a site for marginalized user groups [11]. To benefit from the richness and diversity, a results of continuous interaction among cultures and openness for dialogue, embedded in the living experience, social-structures and cultural-narratives, it is vital that the South Asian HCI community sets a strong agenda to be united to share a vision of HCI that goes beyond the ad-hoc system or service developments from the Global North.

The goal of this workshop is to bring together the community of HCI researchers, designers, and technology makers at CHI 2021 virtually who are interested in research including and beyond development in South Asia. Together, we aim to break the stereotypes and re-imagine the future of $\mathrm{HCI}$ research in the South Asian region, celebrating the diversity, inclusion, and the chaos that comes with it.

More specifically, this workshop has the following objectives:

- To reflect on the current opportunities and challenges in the research being conducted in the South Asian region.

- To explore alternative and empowering vision for research and researchers in South Asia, where research agendas and methods, users groups, and contexts transcend the context of the "user study" to include aspects such as lived experiences, narratives and stories, belongingness and identities, assimilation and co-existence and cultural norms and ethical values.

- To define and identify new agendas and vocabulary to refer to South Asian context, expanding and shifting the current terminology of marginalization, underserved, and developing, to include those that are empowering, equitable, and emancipatory.

We plan to explore various themes during the workshop and exchange ideas and invite conversations (including anecdotes, experiential information, and field stories) during this workshop. We present a few of the exploratory themes that the organizers have discussed and observed, which summarize the perspective of presenting HCI research from South Asia with a broader lens.

\subsection{Including and Beyond Development}

The work situated in the South Asian region is primarily labelled as a contribution from the Global South. As a terminology, Global South has been used by global development organizations to refer to the regions outside Europe and North America which are mostly, not always, low-income and politically and culturally marginalized [4].

The software and services designed in the West are also being utilized by users in South Asia (this includes a significant number of people with higher education, socioeconomic status, device literacy, and access, (e.g.,[1])). The fact that there is a continuous $f u-$ gaads [19] and re-purposing of the devices [20], services and tools that calls for a deeper inspection of the aspirations, opportunities, and capabilities, in order to meaningfully define and design for the South Asian region including and beyond development.

In this regard, researchers, designers, practitioners, and implementers need to expand their lens and view us not only as data points, but as application designers and users, who are basically persons embedded in their social, cultural and political contexts, and as a source of knowledge and an avenue for dissemination for knowledge.

It does not imply that innovation for development should be sidelined. The issues pertaining to accessibility and aging (e.g., [8]), healthcare (e.g., [13, 27]), information literacy, sustainability (e.g., $[9,22]$ ) will continue to pose challenges, increasingly from the perspective of new and emerging technologies (e.g., [15])]. However, they need to be looked as part of the larger social, cultural and political contexts.

\subsection{Considerations for $\mathrm{HCI}$ research}

As HCI researchers, we continuously utilize existing methods, data sources, and theoretical lens for our research. While useful for the Global North, these existing components may not be well-suited to the research questions pursued in other regions of the world. We discuss some of these constraints below in the context of South Asia:

1.2.1 Missing Data. In today's world, where data is the most powerful tool used to utilize and design systems and interactions, the region's missing data can contribute to biased designs and systems. Absence of data either due to the lack of users or lack of consideration of its users and data into design decisions, leads to technology designs that do not work well in the regions of Global South, including our context of interest [3].

However, data is not an inert entity. It is power-laden because the value of data depends on the agencies, intents and perspectives of its collector, interpreter and user, and not necessarily of its generator. Therefore, the agencies, intents and perspectives of data-generator need to critiqued vis-a-vis collector, interpreter and user. In this endeavor, it is important to question the agendas and objectives upon which a set of research questions is based and the lenses that are used to interpret the data.

Thus, we also argue that (a) data from the South Asian region needs to be accounted for and (b) we need to translate designs from these regions - which have been optimized for stricter constraints to other regions of the world and not the other way round. (c) We need to question what are the research questions users would be interested in (d) How do we give agency to the user during data interpretation.

1.2.2 Methods. The existing pool of HCI research methods have been designed and developed with communities which are at times complementary if not unique to the populations of the South Asia. Thus, at times, HCI research and design strategies prove ill-fitted for these contexts [24]. For example, a commonly referred method of Participatory Design where the communities or end-users are engaged as participants of the design process might not be successful in regions where communities feel uncomfortable about sharing their experiences due to power-distance and cultural-disconnect 
between the users and researchers [21] or are unsure of the possibilities with new design [25]. Similarly, recent uptake of online research methods where participants are expected to communicate and participate online might also be not possible in emerging regions. Thus, we argue that the HCI research community needs to look beyond translation of methods into resource constrained environments, for understanding and refinement of methods in these regions and the implications of these methods in new situations.

1.2.3 User Groups. The region has wide varieties of geographical, political, socio-cultural, religious, economic, and linguistic difference all of which form the colorful and vibrant community within and outside these countries. However, the region is also defined by shared history and culture. Thus, expanding the lens of the user groups around and beyond the development will also enable us to see the similarities and differences and design for the cross-cultural and intersectional nature of these communities. Such a broader lens will help us as researchers see how we might be mutually beneficial and view the users of this region beyond consumers of technology including acceptance and appreciation of the ground-up innovations of its inhabitants.

1.2.4 Support. The conversation of what constitutes interesting research has gained recent attention in the HCI field [16]. The $\mathrm{HCI}$ research is mostly supported by technology giants from the Global North (including Microsoft research, IBM research, and Google research) who study users from South Asia under the label of 'emergent users' or 'next billion users' [6]. This labelling further promotes and contributes to the one-dimensional identity of the work from South Asia, i.e., of "for development." Thus, in order to promote and diversify research from this region, we urge the conversation around providing support and mentorship along with funding research, researchers, and ideas outside of what is typically development work.

\section{ORGANIZERS:}

Within their various works, all of the organizers of this workshop belong to and/or have an experience of working in the South Asia on the problems including, at the intersection, and beyond the development.

- Pranjal Jain is the Co-founder of theUXWhale, India. He started Srishti SIGCHI Chapter in 2018. His research interest focuses on understanding social implication of algorithm bias and data privacy.

- Samia Ibtasam is a Ph.D. student in Computer Science \& Engineering at the University of Washington, Seattle. She works on on devising tools and frameworks to increase technological and financial inclusion. Previously, she was a faculty member and founding Co-Director of Innovations for Poverty Alleviation Lab (IPAL) at Information Technology University in Pakistan.

- Sumita Sharma is postdoctoral researcher at the INTERACT Research Unit, University of Oulu, Finland. Her research focuses on empowerment, inclusion, and accessibility through technology use, design, and making with and for diverse groups of children.
- Nilavra Bhattacharya is a Ph.D. student in the School of Information at the University of Texas at Austin. He studies how people find, evaluate, and consume information online, and how to improve the overall Information eXperience.

- Anupriya Tuli is a Ph.D. student in Human-Centred Design at IIIT-Delhi, India. Her work focuses on human-centred computing and healthcare for development, particularly on feminist perspectives on designing technologies for women's advocacy and works extensively with practitioners and NGOs in the field.

- Dilrukshi Gamage is a Ph.D. student in the field of HCI and Education Technology studying at University of Moratuwa, Sri Lanka. She is the founder and the chair for Colombo SIGCHI chapter in Sri Lanka.

- Dhruv Jain is a Ph.D. student in Computer Science \& Engineering at the University of Washington, Seattle. His research focuses on AI and accessibility, particularly to enhance sound awareness for people who are $\mathrm{d} /$ Deaf and hard of hearing.

- Rucha Tulaskar is a lead User Experience researcher and designer at Continuum Managed Services, India with around $19+$ years of professional experience. She is chair of ACM SIGCHI Mumbai Chapter, India.

- Priyank Chandra is Postdoctoral Fellow in Computer Science, and soon to be Assistant Professor at the Faculty of Information, at the University of Toronto. He is interested in designing for communities at the margins of society, informality, and technology and development.

- Lubna Razaq is a faculty member at the Information Technology University, Lahore, Pakistan, and is the founding director for FinTech Center at the Information Technology University.

- Rahat Jahangir Rony is a Graduate Research Assistant in Electrical and Computer Engineering at North South University. His research focuses on HCI, ICTD and Systems. He is the treasurer of Dhaka ACM SIGCHI Chapter (BanglaCHI), Bangladesh.

- Deepak Ranjan Padhi is a Ph.D. student at IDC School of Design,Indian Institute of Technology, Bombay, India. His research focuses on HCI4D and design of Education Technologies for low-literate communities. He is the treasurer of IIT Bombay ACM SIGCHI Student Chapter, India.

- Mohit Jain is a Senior Researcher at Microsoft Research India. His work focuses on healthcare, accessibility and conversational agents.

- Suleman Shahid is an Assistant Professor in Computer Science at Lahore University of Management Science (LUMS), Lahore, Pakistan.

- Nova Ahmed is an Associate Professor, Department of Electrical and Computer Engineering, North South University, Bangladesh.

- Devanuj Balkrishan is a Associate Professor at JK Lakshmipat University, India. His research interests are primarily oriented towards Emergent Users (Next Billion Users).

- Pushpendra Singh is a Professor in the Dept. of Computer Science and Engineering and the Dept. of Human-Centered Design, IIIT-Delhi, India. He works at the intersection of 
HCI, data analytics, and mobile systems and applications with a focus on societal problems, primarily in the areas of Healthcare and Education.

\section{PRE-WORKSHOP PLANS AND WEBSITE:}

We will invite applications from researchers, designers, practitioners, and technology makers, including undergraduate and early career researchers working in the context of South Asia.

The workshop will be conducted online. We will use Zoom for the interaction and presentations, which will be broadcast via YouTube. The repositories, in form of video-recordings and documents, will be preserved and hosted on our website for a wider accessibility. All relevant information about the workshop will be updated and preserved on the website. To include individuals with limited experience in writing scientific articles, we intend to use an open-ended survey administered via Google forms, instead of a position paper. The survey questions will include:

- Lexicon: What are some keywords and phrases that you use to define your research/design activity? Which among them should (i) be changed, (ii) feels limiting, (iii) works as-is. Additionally, what keywords and phrases that should be added to the domain?

- (Research) Questions: Mention up to three problems/(research) questions in your area that needs to be solved. Why do you think these questions or problems are important and worth solving? Who stands to benefit from solving those problems? Do you think these problems will be relevant in the next decade? Why?

- Methods: What are some (research) approaches, tools, or methods that you regularly use in your work, or have employed in the past? Which ones were useful and which ones were not?

- Challenges: What are some of the major barriers that you face in your research/design/development work? This may include challenges in conducting user studies, technology adaptation problems, barriers in reporting and publishing results, financial barriers in information seeking and/or attending research and outreach events (conferences and related venues).

- Select sub-theme(s): Please select one or more of the following sub-themes that you wish to engage in a discussion at the workshop (pertaining to the South Asian region).

The sub themes include (but are not limited to): accessibility, healthcare, (HCI) education, information literacy, development, design, application, design futuring, and more.

- Sharing experience(s): Would you like to share an experience, field story, observation, or anecdote with other participants during the workshop, that was a learning experience for you?

- Expectations from the workshop: What are you hoping to get out of attending this workshop?

Each attendee will submit their survey response. Organizers will perform a thematic analysis on the responses to identity themes for discussion at the workshop. To help organizers group participants, authors may be asked to expand on their survey answers in the period between acceptance and the week preceding the workshop.
At the same time, before the workshop, the organizing team will provide opportunities for participants to contribute to broader topics and ideas in a living document. This will gather a community conversation around the topics of interest in the South Asian region. We will post this information on the workshop website as well. Participants would also be asked to join the Slack channel where they can introduce themselves and know each other interest areas.

Website and Twitter: Details about the workshop, including key dates, will be available via the workshop website at http:// hci4south.asia and twitter https://twitter.com/HCI4SouthAsia. This website and twitter handle will act as the information catalyst in the weeks leading up to the workshop.

\section{WORKSHOP STRUCTURE:}

We will hold a half-day workshop virtually comprising three sessions with activities summarized in Table 1 over the Zoom platform. In these sessions, we will reflect on the past and re-imagine the future, considering questions loosely based on design futuring $[7]^{1}$

- Session I: Reflecting on now (individual, all participants): Participants and organizers (as agreed in the preworkshop activity with several open slots) share one experience (in two minutes each) on conducting research in South Asia. This can include: Reflecting on a challenge, negative, or positive experience; Posing a question to/for the group, along with the rationale or thoughts behind their question; Sharing a vision, hope, or desire for their future research; Highlighting an identified 'need for change'; Other messages, experiences, "open mic." These two-minute presentations, either presented using a previously shared 1-slide or free presentation format, are not thematically organized. Instead, they are diverse, inclusive, varied, and unstructured.

- Session II: Ideating for the future (small groups of participants): Participants are divided into small teams of 3-5, based on their selected sub-theme (in the pre-workshop activity), to spend 30 minutes to discuss:

- The now: Taking stock of the past and present, considering what is currently happening in their specific sub-theme globally and South Asia.

- Future vision: If all things are possible in the future, where we want to be in the future and what are some utopian or ideal future scenarios for researchers and/or for conducting research.

- Approach: What are the opportunities and/or challenges to get from the now to the future vision.

- Drivers: Identifying who is/are responsible to get us there and why and how.

- Progress: Formulating measurable ways to know that the progress is being made to realize the vision.

Participants can use mind-maps, google docs, or slides (or other online collaborative tools) to document their ideas and answers. Teams with the same sub-theme join the same breakout rooms and present and discuss their insights. These

\footnotetext{
${ }^{1}$ The organizers have expertise and experience in conducting design futuring workshops: https://sigchimumbai.acm.org/wp/blog/2020/06/04/27th-june-12th-acm-sigchimumbai-chapter-virtual-meet/
} 


\begin{tabular}{ll}
\hline Time & Activity \\
\hline 11:00-11:15 & Welcome: Introduction from the organizing committee \\
11:15 - 12:15 & Session I: Reflecting on now (individual, all participants) \\
12:15 - 12:30 & Break \\
12:30 - 13:30 & Session II: Ideating for the future (small groups of participants) \\
13:30 - 14:45 & Break \\
14:45 - 15:15 & Session III: Reflections and Synthesis (small groups, followed by all attendees) \\
15:15 - 15:25 & Break \\
15:25-16:00 & Closing: Next Steps and Final Remarks \\
\hline
\end{tabular}

Table 1: Proposed Agenda (half day)

discussions will also be summarized using an online collaborative tool for archival purposes.

- Session III: Reflections and Synthesis (small groups, followed by the whole group): Participants will perform an activity for defining shared vision(s), mission(s), and identities for HCI activities in South Asian

30 minutes: In the thematic small groups (same as in Session II), participants define one or two vision statements, and identify ways to realize the vision, and thus define their mission.

60 minutes: A representative from each subgroup presents a two-minute summary of their discussion to all the attendees. (This will be simultaneously appended in the Living Document by a moderator. ) Next, we will hold a moderated discussion on refining the visions while building on the collectively generated themes (collected in the Living Document).

\section{POST-WORKSHOP PLANS:}

We aim to achieve a three-fold outcome from the workshop:

(1) South Asian Community Creation: First, we hope to identify and create a community of HCI researchers, designers, practitioners, and technology makers engaged in work with and within South Asia. Since this will be the first workshop of its kind, we will form a collective vision of the community about their future agendas, aspirations, and challenges beyond development work. The participants will also be invited to join our mailing list to receive updates about upcoming events and initiatives. The identified vital themes from the workshop discussion, brainstorming, and design activity will be available in the form of the living document for further building and improving by the community.

(2) Broader HCI Community Discussion: Second, the content from the living document collected before and during the workshop, coupled with workshop conversations will result in initiating an HCI community-wide discussion reflecting plans and agendas suggested and shaped by our participants, representing the South Asian HCI community. This community discussion will contribute in (re)shaping our future workshop agendas and sustaining the ongoing conversation beyond the workshop.

(3) Publication(s): Finally, we have strong aims to produce one or more publication(s) to present new themes, principles, and visions for conducting human-centered research and design in South Asia. The publication venue(s) will be determined according to the number of generated outcomes (themes, principles, etc.): a shorter list may result in an article for Communications of the ACM or ACM Interactions; or other related venues. Depending on the workshop outcome, workshop participants will either be acknowledged for their contribution to this publication(s), or be invited for co-authorships. Participants will also be strongly encouraged to publish the results of their discussions with others they have connected with through the workshop. The organizers will as well work with interested participants from the South Asian region to provide writing support as required.

Besides these three aims, we will also identify additional pathways to outputs that may benefit participants directly, in particular those that may have an impact beyond the $\mathrm{CHI}$ community. Participants will be asked to report any resulting publications or other outcomes so that the organizers can send relevant updates.

\section{CALL FOR PARTICIPATION:}

We aim to build "HCI4SouthAsia", a strong community of HCI researchers and practitioners who work/will work in the South Asian region (i.e., in and around the countries Afghanistan, Bangladesh, Bhutan, India, Maldives, Nepal, Pakistan, and Sri Lanka). As a first step, we are organizing a half-day virtual workshop over the Zoom platform at CHI 2021, titled: "From the Margins to the Centre: Defining New Mission and Vision for HCI Researchers in South Asia”.

The primary objective of the workshop is to provide an international platform to showcase - to the "center" - the research (including and beyond development), challenges, experiential information, and field stories, from (marginal) communities across the South Asian Region, and help to collectively build a vision for the community. We invite participants to join our hybrid workshop either in-person or remotely. The half-day workshop will include three activity-based design sessions encompassing reflection on the past, building a vision, and proposing pathways for realizing the vision.

Interested participants are invited to fill a survey to share details about their research, methods, preliminary or prominent results from their work, and their aspirations from the workshop. Participants will be accepted based on how their responses align with the workshop theme. The last day to apply is February 21, 2020. More details about the workshop can be found at http://hci4south.asia and https://twitter.com/HCI4SouthAsia.

\section{REFERENCES}

[1] Pawan Agarwal. 2007. Higher education in India: Growth, concerns and change agenda. Higher education quarterly 61, 2 (2007), 197-207. 
[2] Zane L Berge and Lin Muilenburg. 2013. Mobile learning games for low-income children in India: Lessons from 2004-2009. Routledge, New York and London, 655-666.

[3] Jenna Burrell and Kentaro Toyama. 2009. What constitutes good ICTD research? Information Technologies \& International Development 5, 3 (2009), pp-82.

[4] Nour Dados and Raewyn Connell. 2012. The global south. Contexts 11, 1 (2012), $12-13$.

[5] Nicola Dell and Neha Kumar. 2016. The Ins and Outs of HCI for Development. In Proceedings of the 2016 CHI Conference on Human Factors in Computing Systems (San Jose, California, USA) (CHI '16). Association for Computing Machinery, New York, NY, USA, 2220-2232

[6] Devanuj and Anirudha Joshi. 2013. Technology Adoption by 'emergent' Users The User-Usage Model. In Proceedings of the 11th Asia Pacific Conference on Computer Human Interaction (Bangalore, India) (APCHI '13). Association for Computing Machinery, New York, NY, USA, 28-38.

[7] Anthony Dunne and Fiona Raby. 2013. Speculative everything: design, fiction, and social dreaming. MIT press, Cambridge, USA.

[8] Anita Ghai and Swaminathan Manohar Reddy, Vidhya Yella. 2020. Assistive Technology Research and Disability Studies in the Global South: the Need for Synergy. https://bit.ly/2H8hPvV.

[9] Mohit Jain, Ankit Agrawal, Sunil K. Ghai, Khai N. Truong, and Deva P. Seetharam. 2013. "We Are Not in the Loop": Resource Wastage and Conservation Attitude of Employees in Indian Workplace. In Proceedings of the 2013 ACM International foint Conference on Pervasive and Ubiquitous Computing (Zurich, Switzerland) (Ubi Comp '13). Association for Computing Machinery, New York, NY, USA, 687-696.

[10] Konstantinos Kazakos, Siddhartha Asthana, Madeline Balaam, Mona Duggal, Amey Holden, Limalemla Jamir, Nanda Kishore Kannuri, Saurabh Kumar Amarendar Reddy Manindla, Subhashini Arcot Manikam, GVS Murthy, Papreen Nahar, Peter Phillimore, Shreyaswi Sathyanath, Pushpendra Singh, Meenu Singh, Pete Wright, Deepika Yadav, and Patrick Olivier. 2016. A Real-Time IVR Platform for Community Radio. In Proceedings of the 2016 CHI Conference on Human Factors in Computing Systems (San Jose, California, USA) (CHI '16). Association for Computing Machinery, New York, NY, USA, 343-354.

[11] Shaista E Khilji. 2012. Editor's perspective: does South Asia matter? Rethinking South Asia as relevant in international business research. South Asian fournal of Global Business Research 1, 1 (2012), 8-21.

[12] Neha Kumar, Naveena Karusala, Azra Ismail, and Anupriya Tuli. 2020. Taking the Long, Holistic, and Intersectional View to Women's Wellbeing. ACM Trans. Comput.-Hum. Interact. 27, 4, Article 23 (July 2020), 32 pages.

[13] Eric C. Larson, Mayank Goel, Morgan Redfield, Gaetano Boriello, Margare Rosenfeld, and Shwetak N. Patel. 2013. Tracking Lung Function on Any Phone. In Proceedings of the 3rd ACM Symposium on Computing for Development (Bangalore India) ( $A C M D E V$ '13). Association for Computing Machinery, New York, NY USA, Article 29, 2 pages.

[14] Indrani Medhi Thies. 2017. SIGCHI Social Impact Award Talk - Designing for Low-Literate Users. In Proceedings of the 2017 CHI Conference Extended Abstracts on Human Factors in Computing Systems (Denver, Colorado, USA) (CHI EA '17). Association for Computing Machinery, NY, USA, 8-9.

[15] Indrani Medhi Thies, Nandita Menon, Sneha Magapu, Manisha Subramony, and Jacki O'Neill. 2017. How Do You Want Your Chatbot? An Exploratory Wizard-ofOz Study with Young, Urban Indians. In Human-Computer Interaction - INTERACT 2017, Regina Bernhaupt, Girish Dalvi, Anirudha Joshi, Devanuj K. Balkrishan, Jacki O’Neill, and Marco Winckler (Eds.). Springer International Publishing,
Cham, 441-459.

[16] Ihudiya Finda Ogbonnaya-Ogburu, Angela D.R. Smith, Alexandra To, and Kentaro Toyama. 2020. Critical Race Theory for HCI. In Proceedings of the $2020 \mathrm{CHI}$ Conference on Human Factors in Computing Systems (CHI '20). Association for Computing Machinery, New York, NY, USA, 1-16.

[17] Deepak Ranjan Padhi, Anirudha Joshi, Abhishek Shrivastava, and Rucha Tulaskar. 2018. Hierarchy or List? Comparing Menu Navigation by Emergent Users. In Proceedings of the 9th Indian Conference on Human Computer Interaction (Bangalore, India) (IndiaHCI'18). Association for Computing Machinery, New York, NY, USA, 29-34.

[18] Divya Ramachandran, John Canny, Prabhu Dutta Das, and Edward Cutrell. 2010. Mobile-Izing Health Workers in Rural India. In Proceedings of the SIGCHI Conference on Human Factors in Computing Systems (Atlanta, Georgia, USA) (CHI '10). Association for Computing Machinery, New York, NY, USA, 1889-1898.

[19] Nimmi Rangaswamy and Melissa Densmore. 2013. Understanding Jugaad: ICTD and the Tensions of Appropriation, Innovation and Utility. In Proceedings of the Sixth International Conference on Information and Communications Technologies and Development: Notes - Volume 2 (Cape Town, South Africa) (ICTD '13). Association for Computing Machinery, New York, NY, USA, 120-123.

[20] Nimmi Rangaswamy and Nithya Sambasivan. 2011. Cutting Chai, Jugaad, and Here Pheri: towards UbiComp for a global community. Personal and Ubiquitous Computing 15, 6 (2011), 553-564.

[21] Samia Razaq, Amna Batool, Umair Ali, Muhammad Salman Khalid, Umar Saif, and Mustafa Naseem. 2016. Iterative Design of an Immunization Information System in Pakistan. In Proceedings of the 7th Annual Symposium on Computing for Development (Nairobi, Kenya) (ACM DEV '16). Association for Computing Machinery, New York, NY, USA, Article 9, 10 pages.

[22] Yedendra B. Shrinivasan, Mohit Jain, Deva P. Seetharam, Abhishek Choudhary, Elaine M. Huang, Tawanna Dillahunt, and Jennifer Mankoff. 2013. Deep Conservation in Urban India and Its Implications for the Design of Conservation Technologies. In Proceedings of the SIGCHI Conference on Human Factors in Computing Systems (Paris, France) (CHI '13). Association for Computing Machinery, New York, NY, USA, 1969-1978.

[23] Sharifa Sultana, Syed Ishtiaque Ahmed, and Susan R. Fussell. 2019. "Parar-Daktar Understands My Problems Better": Disentangling the Challenges to Designing Better Access to Healthcare in Rural Bangladesh. Proc. ACM Hum.-Comput. Interact. 3, CSCW, Article 168 (Nov. 2019), 27 pages.

[24] Sharifa Sultana, François Guimbretière, Phoebe Sengers, and Nicola Dell. 2018. Design within a patriarchal society: Opportunities and challenges in designing for rural women in bangladesh. In Proceedings of the 2018 CHI Conference on Human Factors in Computing Systems. Association for Computing Machinery, New York, NY, 1-13.

[25] Kentaro Toyama. 2018. From needs to aspirations in information technology for development. Information Technology for Development 24, 1 (2018), 15-36.

[26] Anupriya Tuli, Shruti Dalvi, Neha Kumar, and Pushpendra Singh. 2019. "It's a Girl Thing”: Examining Challenges and Opportunities around Menstrual Health Education in India. ACM Trans. Comput.-Hum. Interact. 26, 5, Article 29 (July 2019), 24 pages.

[27] Edward Jay Wang, Junyi Zhu, Mohit Jain, Tien-Jui Lee, Elliot Saba, Lama Nachman, and Shwetak N. Patel. 2018. Seismo: Blood Pressure Monitoring Using Built-in Smartphone Accelerometer and Camera. In Proceedings of the $2018 \mathrm{CHI}$ Conference on Human Factors in Computing Systems (Montreal QC, Canada) (CHI '18). ACM, New York, NY, USA, 425:1-425:9. 